\title{
A finite cancellative semigroup is a group
}

\author{
Open Mathematics Collaboration*广
}

August 4, 2020

\begin{abstract}
We prove the proposition addressed in the title of this paper.
\end{abstract}

keywords: finite cancellative semigroup, group theory, abstract algebra

The most updated version of this paper is available at https://osf.io/34vbp/download

\section{Notation \& Definition}

1. $[1,2]$

2. $\mathcal{S}$ = finite semigroup

(finite set + binary operation + associative)

3. $\forall x, y, z \in \mathcal{S}:(z x=z y) \rightarrow(x=y) \quad$ left-cancellative

4. $\forall x, y, z \in \mathcal{S}:(x z=y z) \rightarrow(x=y) \quad$ right-cancellative

5. $((\mathcal{S}=$ left-cancellative $) \wedge(\mathcal{S}=$ right-cancellative $)) \rightarrow(\mathcal{S}=$ cancellative $)$

*All authors with their affiliations appear at the end of this paper.

†Corresponding author: mplobo@uft.edu.br | Join the Open Mathematics Collaboration 


\section{Proposition}

6. A finite cancellative semigroup is a group. [1]

\section{Proof 1}

7. Proposition: All finite semigroups are periodic, i.e., all elements of a finite semigroup are periodic.

8. From (2) and (7), $\mathcal{S}$ is periodic.

9. Let $x \in \mathcal{S}$ arbitrary.

10. Since $x^{k}=x^{\ell}$ for $k<\ell$, then $x^{\ell-k}=1_{\mathcal{S}}$, so $\mathcal{S}$ has identity.

11. Let $y \in S$ arbitrary, then $y^{n}=1_{S}$ for some $n \in \mathbb{N}=\{1,2,3, \ldots\}$.

12. $y^{n}=y^{n-1} y=y y^{n-1}=1_{S}$

13. So, $y^{n-1}$ is an inverse (left and right).

14. Therefore, $\mathcal{S}$ is a group.

\section{Proof 2}

15. $T(\mathcal{S})=$ transformation semigroup

16. From Cayley's theorem, $\mathcal{S} \cong T(\mathcal{S})$.

17. Since $\mathcal{S}$ is finite and cancellative, the elements in $T(\mathcal{S})$ are permutations.

18. Any finite semigroup of permutations is a group.

19. Thus $\mathcal{S} \cong$ group.

20. Therefore, $\mathcal{S}$ is a group. 


\section{Final Remarks}

21. $(\mathcal{S}=$ finite cancellative semigroup $) \rightarrow(\mathcal{S}=$ group $)$

\section{Open Invitation}

Review, add content, and co-author this paper [3,4]. Join the Open Mathematics Collaboration (https://bit.ly/ojmp-slack). Send your contribution to mplobo@uft.edu.br.

\section{Open Science}

The latex file for this paper together with other supplementary files are available [5].

\section{Ethical conduct of research}

This original work was pre-registered under the OSF Preprints [6], please cite it accordingly [7]. This will ensure that researches are conducted with integrity and intellectual honesty at all times and by all means.

\section{Acknowledgement}

+ Center for Open Science https://www.cos.io

+ Open Science Framework https://osf.io 


\section{References}

[1] Cain, Alan J. Nine Chapters on the Semigroup Art. AJC Porto \& Lisbon, 2020. http://www-groups.mcs.standrews.ac.uk/\%7ealanc/teaching/m431

[2] Lobo, Matheus P. "Open Mathematics Knowledge Base." OSF Preprints, 13 May 2020. https://doi.org/10.31219/osf.io/evq8a

[3] Lobo, Matheus P. "Microarticles." OSF Preprints, 28 Oct. 2019. https://doi.org/10.31219/osf.io/ejrct

[4] Lobo, Matheus P. "Simple Guidelines for Authors: Open Journal of Mathematics and Physics." OSF Preprints, 15 Nov. 2019. https://doi.org/10.31219/osf.io/fk836

[5] Lobo, Matheus P. "Open Journal of Mathematics and Physics (OJMP)." OSF, 21 Apr. 2020. https://doi.org/10.17605/osf.io/6hzyp

[6] COS. Open Science Framework. https://osf.io

[7] Lobo, Matheus P. "A Finite Cancellative Semigroup Is a Group." OSF Preprints, 28 June 2020. https://doi.org/10.31219/osf.io/34vbp

\section{The Open Mathematics Collaboration}

Matheus Pereira Lobo (lead author, mplobo@uft.edu.br) $)^{1,2}$

https://orcid.org/0000-0003-4554-1372

${ }^{1}$ Federal University of Tocantins (Brazil)

${ }^{2}$ Universidade Aberta (UAb, Portugal) 